\title{
ACUTE CAFFEINE ADMINISTRATION IMPACT ON WORKING MEMORY-RELATED BRAIN ACTIVATION AND FUNCTIONAL CONNECTIVITY IN THE ELDERLY: A BOLD AND PERFUSION MRI STUDY
}

\author{
S. HALLER, ${ }^{a *}$ C. RODRIGUEZ, ${ }^{b}$ D. MOSER, ${ }^{a}$ S. TOMA, ${ }^{b}$ \\ J. HOFMEISTER, ${ }^{b}$ I. SINANAJ, ${ }^{b}$ D. VAN DE VILLE, ${ }^{c, d}$ \\ P. GIANNAKOPOULOS ${ }^{b}$ AND K.-O. LOVBLAD ${ }^{a}$ \\ ${ }^{a}$ Department of Imaging and Medical Informatics, University \\ Hospitals of Geneva and Faculty of Medicine of the University \\ of Geneva, Switzerland \\ ${ }^{\mathrm{b}}$ Department of Mental Health and Psychiatry, University Hospitals \\ of Geneva and Faculty of Medicine of the University of \\ Geneva, Switzerland \\ ${ }^{\mathrm{c}}$ Department of Radiology and Medical Informatics, University \\ of Geneva, Switzerland \\ d Institute of Bioengineering, Ecole Polytechnique Fédérale de \\ Lausanne, Switzerland
}

\begin{abstract}
tex as well as the occipital cortex (visual stimuli) and basal ganglia. The inverse comparison of placebo versus caffeine had no significant difference. Activation strength of the task-relevant-network component correlated with response accuracy for caffeine yet not for placebo, indicating a selective cognitive effect of caffeine. The present findings suggest that acute caffeine intake enhances WM-related brain activation as well as functional connectivity of blood oxygen level-dependent fMRI in elderly individuals. (c) 2013 IBRO. Published by Elsevier Ltd. All rights reserved.
\end{abstract}

Key words: fMRI, BOLD, caffeine, cognition, aging.

\section{INTRODUCTION}

Caffeine is the most widely consumed psychoactive drug with beneficial neurocognitive effects including enhanced attention and memory (Koppelstaetter et al., 2010). Early studies postulated that caffeine's effects on brain activation depend on a complex and potentially regionally variable interaction of neuronal and vascular responses (Laurienti et al., 2003; Koppelstaetter et al., 2010). At the neuronal level, caffeine causes most of its biological effects via the antagonism of all types of adenosine receptors $A 1, A 2 A, A 3$, and $A 2 B$ mainly in the striatal neurons projecting to the basal ganglia (for reviews see Fisone et al. (2004) and Costenla et al. (2010)). Besides this antagonism, it enhances the sensitivity of striatal GABAergic synapses to the stimulation of cannabinoid CB1 receptors and at high concentrations inhibits phosphodiesterases and promotes calcium release from intracellular stores (Rossi et al., 2010). Motor and reinforcing effects of caffeine depend on the ability of caffeine to release pre- and postsynaptic brakes that adenosine imposes on the ascending dopaminergic system. By targeting A1-A2A receptor heteromers in striatal glutamatergic terminals and $A 1$ receptors in striatal dopaminergic terminals, caffeine induces glutamate-dependent and glutamate-independent release of dopamine. It also favors the postsynaptic effect of dopamine by attenuating the antagonistic interactions in the striatal A2A-D2 and A1-D1 receptor heteromers. Arousing properties of caffeine depend on the blockade of multiple inhibitory mechanisms that adenosine, as an endogenous sleep-promoting substance, exerts on the ascending histaminergic and orexinergic systems (Ferre, 2010). Apart from its direct effect on brain cells, caffeine

${ }^{*}$ Corresponding author. Address: Service neuro-diagnostique et neuro-interventionnel DISIM, University Hospitals of Geneva, rue (0)-22-37-23311; fax: + 41-(0)-22-37-27072.

E-mail address: sven.haller@hcuge.ch (S. Haller).

Abbreviations: ASL, arterial spin labeling; BOLD, blood oxygen leve dependent; CBF, cerebral blood flow; CDR, Clinical Dementia Rating scale; DTI, diffusion tensor imaging; fMRI, functional magnetic resonance imaging; FSL, FMRIB's Software Library; GLM, general linear model; MEG, magnetoencephalography; ICs, independent components; PFC, pre-frontal cortex; SMA, supplementary motor area; TICA, tensorial-independent component analysis; VPMC, ventral premotor cortex; WM, working memory. 
has a marked regulatory effect on brain perfusion. Overall, caffeine administration induces a direct vasoconstrictor effect resulting in reduced cerebral blood flow (CBF) via the blockade of $A 1, A 2 A$, and $A 2 B$ subtypes located in cells comprising the neurovascular unit (neurons, astrocytes, and vascular smooth muscle) (Fredholm et al., 1999; Dunwiddie and Masino, 2001). However, the mechanisms surrounding this regulation are by far not elucidated (for review see Pelligrino et al. (2010) and Crunelle et al. (2012)).

Both caffeine-mediated blockade of adenosine receptors and vasoconstriction have direct repercussions on brain connectivity at resting states and during cognitive activation. Several functional magnetic resonance imaging (fMRI) studies documented the decrease of connectivity and global signal amplitude in various cortical areas at resting states (Rack-Gomer et al., 2009; Rack-Gomer and Liu, 2012; Wong et al., 2012). The rare fMRI activation studies used simple perceptual tasks (i.e. auditory oddball, visual stimuli), and suggested the presence of a caffeine-mediated neurovascular uncoupling in young individuals characterized by a significant decrease of CBF with a concomitant increase of evoked (and possibly baseline) oxygen metabolism mainly in frontal areas (Perthen et al., 2008; Griffeth et al., 2011; Diukova et al., 2012). Two fMRI activation studies using a placebo-controlled design and working memory (WM) activation paradigms showed a caffeine-induced blood oxygen leveldependent (BOLD) increase in fronto-parietal areas and a decrease in the thalamus (Koppelstaetter et al., 2008; Klaassen et al., 2013) in young to middle-aged volunteers ranging from 25 to 61 years. Previous functional connectivity studies assessed young adults (Rack-Gomer et al., 2009; Rack-Gomer and Liu, 2012; Wong et al., 2012). To date, no study explored the effects of caffeine on brain activation patterns during highly demanding cognitive tasks in elderly cohorts. However, recent contributions pointed to significant agerelated changes of fMRI activation patterns including higher BOLD signal variability and lower maximal BOLD and flow responses during aging (D'Esposito et al., 2003; Kannurpatti et al., 2010, 2011; Gauthier et al., 2013). In combination with the observation that caffeine has a more pronounced effect on psychomotor and cognitive function in older versus younger participants (Rees et al., 1999), we hypothesized that that caffeinerelated modifications of the BOLD response might be even more pronounced in ageing.

Using a double-blinded, placebo-controlled design, we assessed the impact of caffeine on brain activation during an $n$-back verbal WM task in cognitively preserved elderly participants. Potential effects of caffeine include specific task-related activations, changes in functional connectivity, as well as global vascular effects. The current investigation explores all three potential effects of caffeine: task-related activations using a hypothesisdriven general linear model (GLM) analysis, functional connectivity using data-driven tensorial-independent component analysis (TICA) and direct vascular effects using arterial spin labeling (ASL) perfusion imaging.

\section{EXPERIMENTAL PROCEDURES}

\section{Participants}

The local institutional ethics committee approved this prospective study and all participants gave written informed consent prior to inclusion. We included 24 elderly individuals (mean age $68.8 \pm 4.0$ years, range $62-80$ years, 17 females, $8-11$ years of education). All participants were screened with the Mini Mental State Examination (Folstein et al., 1975), the Lawton's Instrumental Activities of Daily Living (Barberger-Gateau et al., 1992), and the Hospital Anxiety and Depression Scale (Zigmond and Snaith, 1983). Cognitive evaluation was performed as follows: attention (Code, Trail Making Test A), WM (verbal: Digit Span Forward (Wechsler, 1955), visuo-spatial: Corsi Block Tapping (Milner, 1971)), episodic memory (verbal: Buschke Double Memory 48 items (Buschke et al., 1997), visual: Shapes (Baddley et al., 1994)), executive functions (Trail Making Test B (Reitan, 1958)), language (Boston Naming (Kaplan et al., 1983)), visual gnosis (Ghent Overlapping Figures). Subjects were also evaluated with the Clinical Dementia Rating scale (CDR) (Hughes et al., 1982), and only cases with a CDR score of 0 and scores within 1.5 standard deviations of the age-appropriate mean in all other tests were included in the final series. All participants had normal or corrected-to-normal visual acuity, and none reported a history of major medical disorders (cancer, cardiac illness), sustained head injury, psychiatric or neurological disorders, and alcohol or drug abuse. Subjects with regular use of psychotropics, stimulants and B-blockers were excluded. All participants were habitual caffeine consumers taking one to three cups of coffee per day. Participants were instructed to stop consuming caffeine-containing food as from $18 \mathrm{~h}$ the evening prior to the MRI exam. Imaging was performed during the morning of the following day between 9 and $12 \mathrm{~h}$. Structural brain abnormalities were excluded after routine radiological assessment. White matter lesions, presumably related to microvascular leucoencephalopathy, are frequent in the investigated age-group. To limit the impact of this confounding factor, we included only volunteers with a maximum of a Fazekas score of 1 (Fazekas et al., 1987). Moreover, (asymptomatic) acute ischemic lesions were excluded using diffusion tensor imaging (DTI) trace imaging.

\section{WM task}

In a prospective crossover design, each participant had 2 scanning days separated by 1 or 2 weeks. Half of the participants had caffeine at day 1 and placebo at day 2 , and the other half had the inverse order of substances. Medication was applied in a double-blinded way. One capsule (caffeine $200 \mathrm{mg}$ or placebo) was taken per os $30 \mathrm{~min}$ prior to the MR imaging in agreement with previous investigations (Mulderink et al., 2002; Liu et al., 2004; Behzadi and Liu, 2006; Perthen et al., 2008; Rack-Gomer et al., 2009), corresponding to approximately $2-2 \frac{1}{2}$ cups of coffee. 
Participants performed an $n$-back task, a wellestablished WM task in $\mathrm{FMRI}$ (for example, see metaanalysis in (Owen et al., 2005)). Briefly, a sequence of letters was presented visually on an MR compatible canvas in the MRI scanner. In the active 2-back condition, targets are letters that are identical to the letter presented two items ago (e.g. "a $f \mathrm{~h}$ f'). This task has a high demand on WM. In the control condition 0-back, the target is a pre-defined letter (e.g. " $x$ "). This control condition has similar demands on visual processing yet requires only minimal WM. Both conditions are contrasted to evaluate the effect of WM demand in 2-back versus the control condition 0-back. Participants were familiarized with the task demands outside the MRI using a training session. Each run consisted of alternation blocks of $35 \mathrm{~s}$ each for conditions 2-back and 0-back with alternating rest conditions of $15 \mathrm{~s}$ to allow the hemodynamic response to recover from the previous block. Each condition was repeated five times in a pseudo-randomized order. Participants provided response (target versus no target) via an MR compatible response box for targets (33\% of trials) and another button for non-targets. Including an initial fixation period, each run lasted 8-10 min, and each participant performed two runs per day.

\section{MR imaging}

MR imaging was performed on a clinical routine whole body 3.0T MR scanner (TRIO, Siemens medical systems, Erlangen, Germany). Functional imaging implemented a standard echo-planar imaging (EPI) sequence with the following fundamental parameters: whole brain coverage, $74 \times 74$ matrix, 40 slices, voxel size $2.97 \times 2.97 \times 3.0 \mathrm{~mm}^{3}$, echo time (TE) $26 \mathrm{~ms}$, repetition time (TR) $2500 \mathrm{~ms}, 196$ repetitions. An additional 3DT1 sequence was acquired for spatial normalization with the following fundamental parameters: $256 \times 256$ matrix, 176 slices, $1 \times 1 \times 1 \mathrm{~mm}^{3}$, TE $2.3 \mathrm{~ms}$, TR $2300 \mathrm{~ms}$. Additionally, a pulsed arterial spin labeling ( $\mathrm{pASL}$ ) sequence was acquired: $64 \times 64$ matrix, 24 slices, voxel size $3.44 \times 3.44 \times 5 \mathrm{~mm}^{3}$, TE $12 \mathrm{~ms}$, TR $4000 \mathrm{~ms}$, inversion time (TI) $1600 \mathrm{~ms}$. Additional sequences (T2w, DTI, fluid attenuated inversion recovery FLAIR) were acquired and analyzed to rule out concomitant disease.

\section{Statistical analysis}

Statistical analysis was performed in Graphpad Prism, Version 5. After normality testing (D'Agostino \& Pearson's omnibus normality test), the participant's average reaction time and response accuracy for 2-back and 0-back were compared between CAFFEINE versus PLACEBO using repeated-measures $t$-tests.

\section{GLM analysis of task-related activation}

Task-related GLM data processing was carried out using FEAT (FMRI Expert Analysis Tool) Version 5.98, part of FSL (FMRIB's Software Library, www.fmrib.ox.ac.uk/fsl). At the first level, the contrast of 2-back versus 0-back (and the inverse comparison) was calculated separately for each run of each participant. At the second level, the intra-participant difference in the two runs of CAFFEINE versus PLACEBO (and the inverse comparison) was assessed individually. At the third level, the group average across all 24 participants was calculated. Higher-level analysis was carried out using a fixed effects model, by forcing the random effects variance to zero in FLAME (FMRIB's Local Analysis of Mixed Effects) (Beckmann et al., 2003; Woolrich et al., 2004; Woolrich, 2008). Z (Gaussianised T/F) statistic images were thresholded using clusters determined by $Z>2.3$ and a corrected cluster significance threshold of $p=0.05$. Anatomic location of the activation clusters was determined using "atlasquery", part of FSL, and the Harvard-Oxford Cortical Structural Atlas.

\section{TICA analysis of functional connectivity}

Analysis was carried out using TICA (Beckmann and Smith, 2005) as implemented in MELODIC (Multivariate Exploratory Linear Decomposition into Independent Components) Version 3.10, part of FSL (FMRIB's Software Library, www.fmrib.ox.ac.uk/fsl). The following data pre-processing was applied: masking of non-brain voxels; voxel-wise de-meaning of the data; normalization of the voxel-wise variance. Pre-processed data were whitened and projected into a 40-dimensional subspace using Principal Component Analysis. The whitened observations were decomposed into sets of vectors, which describe signal variation across the temporal domain (time-courses), the session/participant domain and across the spatial domain (maps) by optimizing for non-Gaussian spatial source distributions using a fixed-point iteration technique (Hyvarinen, 1999). Estimated Component maps were divided by the standard deviation of the residual noise and thresholded by fitting a mixture model to the histogram of intensity values (Beckmann and Smith, 2004). The participant S-modes (a measure of the activation strength) of each of the 40 independent components (ICs) were post hoc compared between CAFFEINE versus PLACEBO implementing repeated-measure $t$-tests and Bonferroni's correction for multiple comparisons. For the taskrelevant ICs and for both CAFFEINE and PLACEBO conditions, the S-modes were then correlated with the behavioral response accuracy of the demanding 2-back task. The statistical significance of Pearson's correlation was then assessed using a standard parametric test procedure.

\section{Analysis of ASL perfusion}

The reconstructed relCBF ASL perfusion images were spatially normalized using a linear transformation equivalent to pre-processing of the functional MRI data. We first analyzed the average ASL signal of CAFFEINE versus PLACEBO using a repeated measure $t$-test, followed by a voxel-wise group-level permutation testing in RANDOMISE, part of FSL with threshold-free cluster enhancement (TFCE) (Smith and Nichols, 2009) 
correction for multiple comparisons, considering fully corrected $p$-values $<0.05$ as significant.

\section{RESULTS}

\section{Behavioral data}

There was no significant difference in response accuracy or reaction time for CAFFEINE versus PLACEBO (see Fig. 1). Importantly, all of the cases correctly performed more than $85 \%$ of the 2 -back trials.

\section{GLM analysis of task-related activation}

The average activation of the contrast 2-back versus 0-back yielded strong activations for CAFFEINE and PLACEBO in the established WM network including the

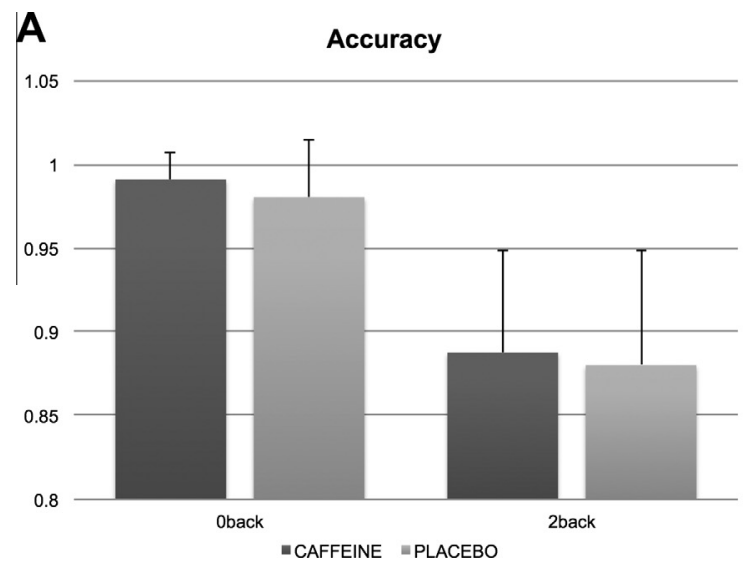

bilateral pre-frontal cortex (PFC), the supplementary motor area (SMA), the ventral and dorsal premotor cortex (vPMC, dPMC) and the parietal cortex. Additional activations were present in the occipital cortex related to the visual stimulus presentation, the basal ganglia and the cerebellum (Fig. 2A, B). The comparison of this contrast for CAFFEINE versus PLACEBO revealed significantly increased BOLD activation in a distributed network including notably the bilateral striatum and to a lesser degree the right middle and inferior frontal gyrus, bilateral insula, the left superior and inferior parietal lobule as well as the cerebellum bilaterally (Fig. 2C). The inverse comparison yielded higher activation for PLACEBO notably in the bilateral superior parietal lobule (Fig. 2D).

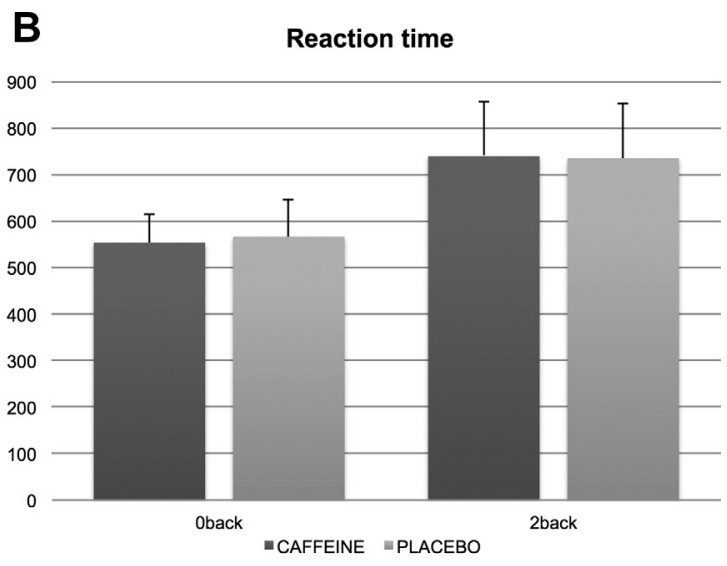

Fig. 1. Illustrates the behavioral response accuracy $(A)$ and reaction time (B) for the 0-back and 2-back conditions. There was no significant difference between CAFFEINE and PLACEBO.

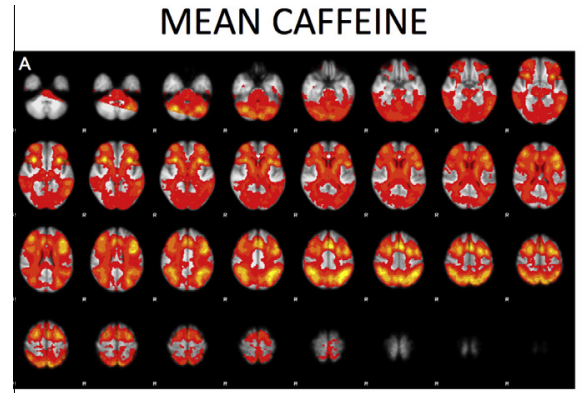

CAFFEINE $>$ PLACEBO

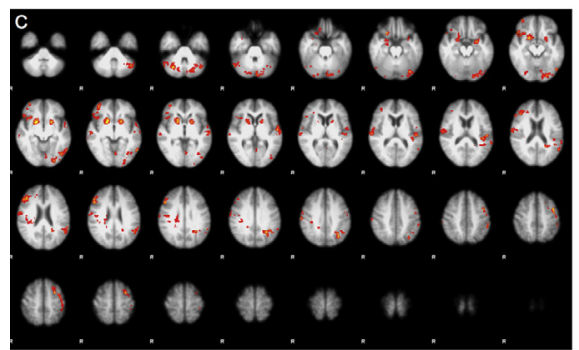

MEAN PLACEBO

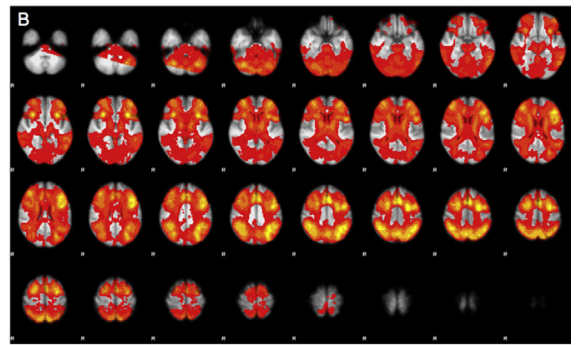

PLACEBO > CAFFEINE

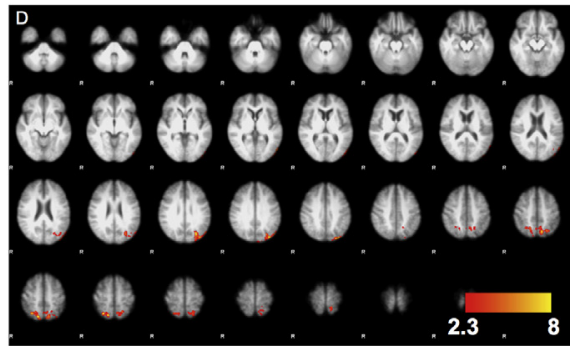

Fig. 2. Illustrates the group average task-related GLM analysis of 2-back versus 0-back for CAFFEINE (A) and PLACEBO (B), involving the established working memory network as well as visual areas related to the stimulus presentation. The contrast of CAFFEINE $>$ PLACEBO (C) yielded significant activations notably in bilateral striatum and to a lesser degree in right-dominant middle and inferior frontal gyrus, bilateral insula and left superior and inferior parietal lobule as well as bilateral cerebellum. The inverse comparison of PLACEBO $>$ CAFFEINE (D) yielded significant activations notably in bilateral superior parietal lobule. Right hemisphere on the left hand side, all clusters were thresholded by $Z>2.3$ and a corrected cluster significance threshold of $p=0.05$. 


\section{TICA analysis of functional connectivity}

Of all 40 ICs, only one IC showed a significant difference $(p<0.05$ corrected) between CAFFEINE versus PLACEBO. The spatial representation of this IC notably includes the established WM network including the PFC, the SMA, the VPMC and the parietal cortex as well as visual areas and the basal ganglia (see Fig. $3 A$ ). The temporal representation of this IC closely resembles the block design ON-OFF of the experimental task (see Fig. 3B). The S-mode (measure of effect size) was $8.2 \%$ higher for CAFFEINE versus PLACEBO (see Fig. 3D). The inverse comparison of PLACEBO versus CAFFEINE yielded no significant differences. There was a significant correlation between individual S-modes of this IC and response accuracy (2-back task) only for CAFFEINE but not for PLACEBO (see Fig. 4).

\section{Analysis of ASL perfusion}

The group average analysis of the ASL perfusion resulted in bilateral symmetric perfusion predominantly in the gray matter (Fig. 5). On average, across the entire gray matter, the ASL signal was reduced by $22.7 \%(p<0.001)$ for CAFFEINE versus PLACEBO. The local voxel-wise comparison of CAFFEINE versus PLACEBO (and inverse) yielded no multiple comparison corrected supra-threshold differences.

\section{DISCUSSION}

Our task-related data analysis revealed higher activation for caffeine versus placebo mainly in the bilateral striatum (an area with high densities of adenosine receptor-positive neurons; (Fisone et al., 2004)), but also in the right middle and inferior frontal gyrus, bilateral insula, and the left superior and inferior parietal lobule. These frontoparietal areas are known to participate in the WM encoding circuit (Klaassen et al., 2013). In the first study that used a very similar activation paradigm in 16 young healthy male participants aged from 25 to 47 , caffeine induced an increased BOLD response in the bilateral medial frontopolar cortex extending to the right anterior cingulate cortex (Koppelstaetter et al., 2008). The effect size in our study was substantially higher than that observed by Koppelstaetter and colleagues. Besides an increased effect of caffeine in ageing, this difference could be explained by a larger sample size (24 versus 16 participants), a higher magnetic field (3 $\mathrm{T}$ versus 1.5 T) and a higher dose of caffeine used in our setting (200 mg versus $100 \mathrm{mg}$ ). More recently, another study assessed the effect of $100 \mathrm{mg}$ of caffeine on WM performance in 20 healthy male participants aged 40-61 during the successful performance of the letter Sternberg task (Klaassen et al., 2013). Caffeine
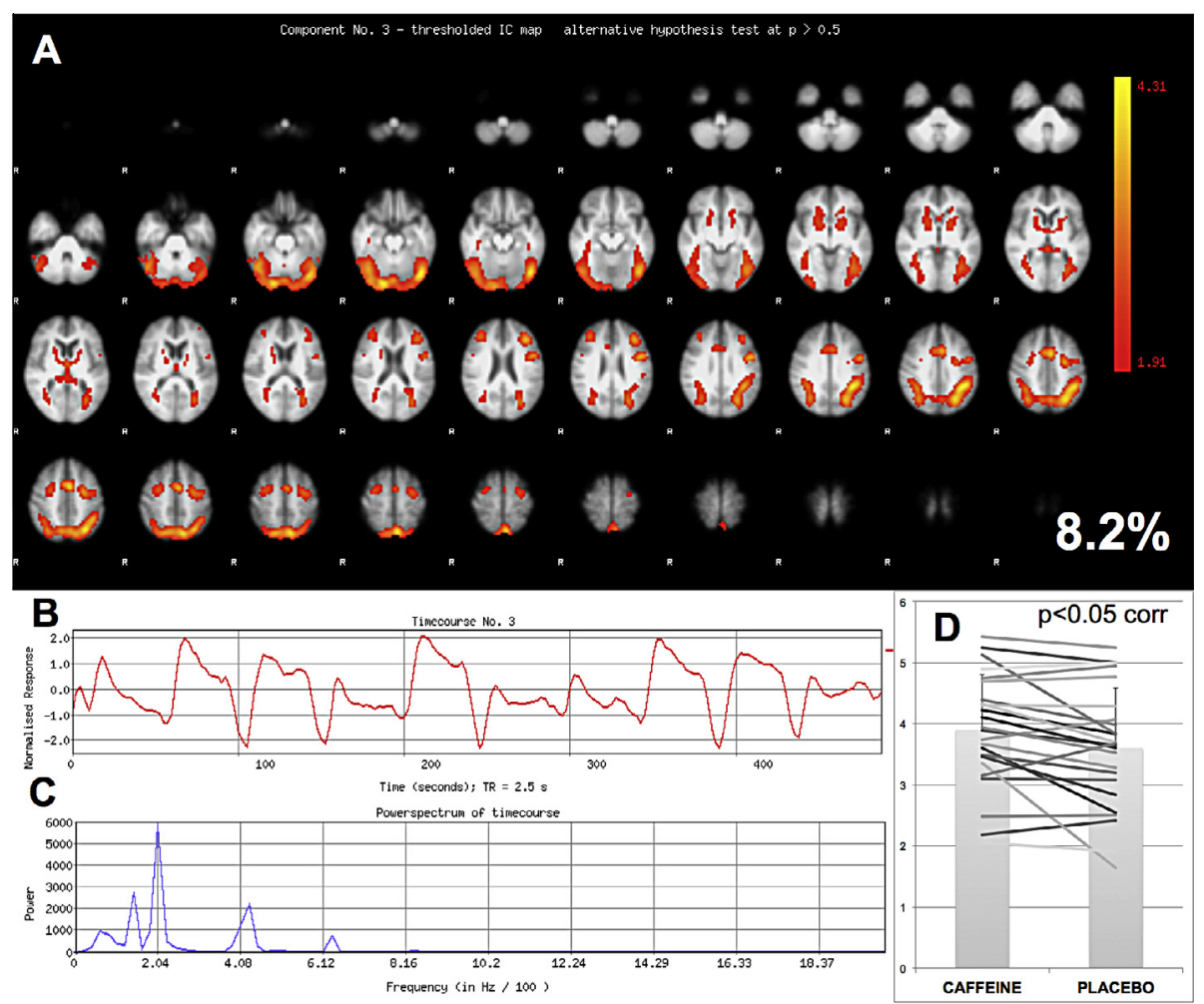

Fig. 3. IC analysis indicates a caffeine-related enhancement in functional connectivity in a working memory task-related network. There was only one component with a significant $(p<0.05$ corrected) difference between CAFFEINE and PLACEBO. The spatial representation of this IC (A) includes the working memory network including pre-frontal cortex (PFC), supplementary motor area (SMA), ventral premotor cortex (VPMC) and parietal cortex. Additional activations are present in the visual areas and the basal ganglia. The temporal representation of this component (B) matches the experimental paradigm. The power spectrum of this component is illustrated in (C). The repeated-measures analysis of this component (D) shows a significant increase in S-mode of $8.2 \%$ for CAFFEINE versus PLACEBO. 


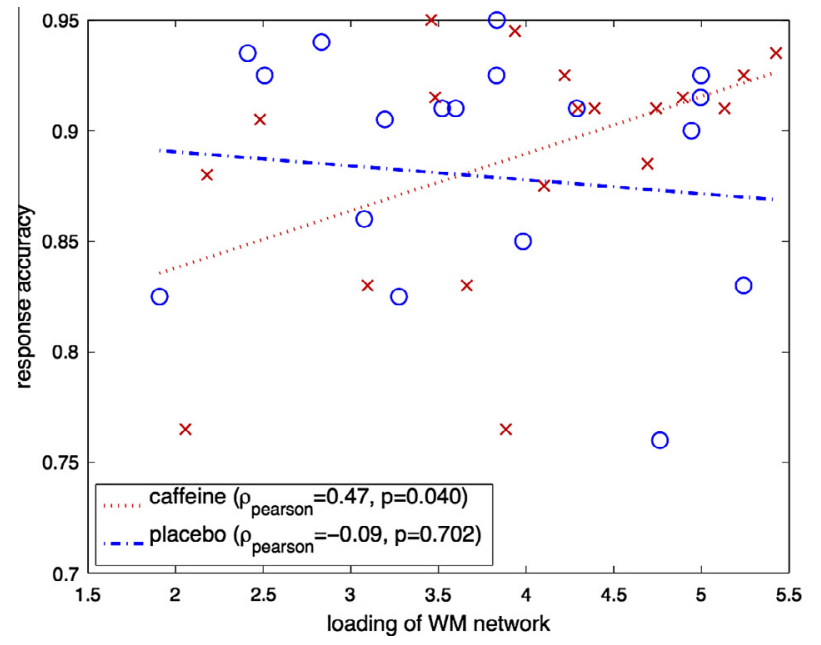

Fig. 4. Correlation between the individual S-modes as a measure of activation strength of the working-memory network and individual behavioral response accuracy is positive only for CAFFEINE but not PLACEBO groups. Red " $x$ " and the dotted red line are individual values and the group-level pearson's fit for CAFFEINE, while blue "o" and the dashed blue line are corresponding individual values and group-level pearson's fit for PLACEBO. (For interpretation of the references to color in this figure legend, the reader is referred to the web version of this article.)

increased load-related activation compared to placebo in the left and right dorsolateral prefrontal cortex during encoding, but decreased load-related activation in the left thalamus during maintenance. Our results in elderly cases confirm previous observations and in particular those of Klaassen et al. (2013) who used a similar WM activation paradigm. However, they also reveal a positive effect of caffeine on the activation of the striatum in old age as well as a decreased activation of the superior parietal cortex bilaterally. Pointing to the importance of the striatum for WM performance in this age group, a recent PET-fMRI study in older cases showed that the striatal dopamine projections to the prefrontal cortex are critical for the integration of WM maintenance process (Landau et al., 2009).

These local increases of BOLD signal are difficult to interpret without a parallel analysis of functional connectivity. In contrast to the above-mentioned studies that applied only task-related GLM analyses, additionally we performed a TICA analysis that made it possible to assess the degree of functional connectivity. Our data reveal a caffeine-related enhancement of functional connectivity in the PFC, VPMC, the SMA, the parietal cortex as well as visual areas (related to the visual stimulus presentation) and the basal ganglia during the highly demanding 2-back task. Importantly, previous fMRI studies reported a caffeine-mediated widespread decrease of functional connectivity at resting states related to its arousing properties. For instance, two recent investigations found that caffeine reduces BOLD functional connectivity and increases its temporal variability in the motor cortex (Rack-Gomer et al., 2009; Rack-Gomer and Liu, 2012). Given the absence of activation paradigm in these studies, it is hard to compare these data with those obtained in the present experimental setting. Another recent study showed that caffeine significantly enhances the detection of the known anti-correlations between the default mode network (DMN) and the task positive network (TPN) (Wong et al., 2012). Subsequently, the WM-load related an increase in functional connectivity after caffeine intake observed here cannot be attributed to a nonspecific effect of this substance. Interestingly, a recent study combined fMRI and magnetoencephalography (MEG) to assess whether changes in resting state fMRI functional connectivity are due to vascular or neural factors (Tal et al., 2013). The functional connectivity changes in fMRI were paralleled by changes in MEG, which directly assesses neuro-electric activity. Consequently, the authors conclude that changes in fMRI functional connectivity have a neural basis.

In conjunction with the GLM data, the present findings support the idea that caffeine acts as an activator of BOLD activation and functional connectivity in WMrelated cortical circuits in elderly individuals. Despite the lack of a global behavioral effect of caffeine during the WM task, we found a significant positive correlation between the activation strength of the working-memory network and the behavioral response accuracy in a 2-

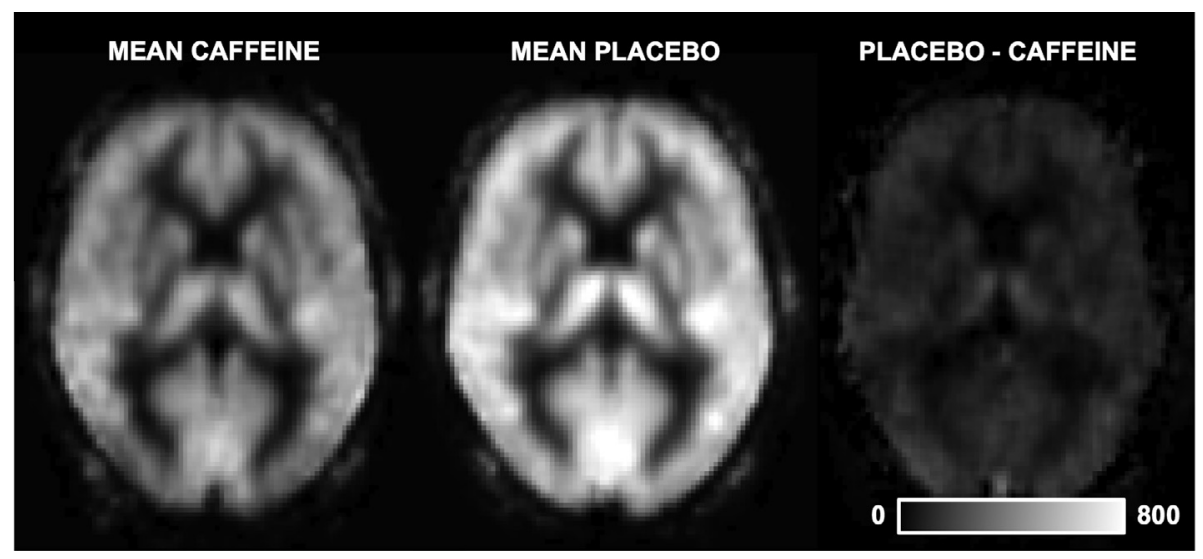

Fig. 5. Average brain perfusion assessed using ASL imaging for CAFFEINE (A), PLACEBO (B), and the difference of PLACEBO minus CAFFEINE (C). All images have the same window levels (0-800 arbitrary units). On average across the gray matter, the global ASL signal is reduced by $22.7 \%$ after CAFFEINE exposure, while there was no supra-threshold local voxel-wise difference between CAFFEINE and PLACEBO. 
back task, only for CAFFEINE yet not for PLACEBO. This observation further points to the specificity of the association between the caffeine-mediated activation of the above-mentioned cortical circuits and WM performance in the elderly. The absence of behavioral changes in response to caffeine exposure despite the measurable enhancement of BOLD activations is consistent with the conclusion of a recent investigation which stated that caffeine should be viewed as a cognitive normalizer rather than a cognitive enhancer (Cunha and Agostinho, 2010) in cognitively normal elderly participants.

As in younger cohorts, caffeine induced a global reduction of baseline perfusion in the range of $20 \%$ (Cameron et al., 1990; Mulderink et al., 2002; Vidyasagar et al., 2013). This effect is specific to caffeine, as for example the same effect was found for caffeine-containing tea, while decaffeinated tea did not reduce cerebral perfusion (Vidyasagar et al., 2013).

As a cerebral vasoconstrictor, caffeine causes an increase in the concentration of deoxyhemoglobin and thus a decrease in the BOLD baseline resting signal by $4.4 \%$, which in turn results in an overall increase in the BOLD contrast by $22-37 \%$ in a visual task (Mulderink et al., 2002). Even usual dietary caffeine consumption significantly interacts with the BOLD response and this is significantly greater in higher dose caffeine consumers (Laurienti et al., 2002). The exact interaction of caffeine on the BOLD response includes a quickening and shortening of the BOLD response in addition to an increase of the BOLD amplitude (Liu et al., 2004), reduction of the initial dip in the visual BOLD response (Behzadi and Liu, 2006) as well as an increase in the linearity of the BOLD response (Liu and Liau, 2010). One could postulate that the observed WM-load related BOLD increase after caffeine ingestion might be partly attributable to its direct vascular effect. However, this is an unlikely scenario since we did not identify local differences in tissue perfusion using a voxel-wise analysis permutation testing.

Strengths of the present study include the double-blind placebo-controlled design, the use of a detailed neuropsychological battery to control for mild cognitive deficits, the exclusion of cases with chronic administration of psychotropic agents, and concomitant analysis of caffeine effects on brain activation, functional connectivity and perfusion using three separate analytical tools. From this viewpoint, this work should be viewed as an additional effort to control the numerous confounders of BOLD fMRI activation after caffeine administration (lannetti and Wise, 2007). There are, however, some limitations that should be carefully considered. Firstly, the limited number of cases did not allow for the distinct exploration of the caffeine impact in the different age groups comprising the elderly population. Secondly, the possible effect of usual caffeine intake on baseline BOLD signal in some of these elderly individuals was not assessed due to the limited sample. Lastly, fMRI data did not provide sufficient information about the temporal dynamics of brain activation during WM processes. Future studies combining $\mathrm{fMRI}$ and EEG activation studies in larger cohorts of well-characterized elderly controls with various doses of caffeine intake are needed in order to clarify the temporal dynamics of brain activation patterns associated with this widely consumed stimulant in old age.

\section{DISCLOSURE}

No conflicts of interest.

Acknowledgement-This work is supported by Swiss National Foundation Grant SNF 3200B0-116193 and SPUM 33CM30124111.

\section{REFERENCES}

Baddley A, Emslie H, Nimmo-Smith I (1994) A test of visual and verbal recall and recognition. Bury St. Edmunds: Thames Valley Test Company.

Barberger-Gateau $\mathrm{P}$, Commenges $\mathrm{D}$, Gagnon $\mathrm{M}$, Letenneur $\mathrm{L}$, Sauvel C, Dartigues JF (1992) Instrumental activities of daily living as a screening tool for cognitive impairment and dementia in elderly community dwellers. J Am Geriatr Soc 40:1129-1134.

Beckmann CF, Smith SM (2004) Probabilistic independent component analysis for functional magnetic resonance imaging. IEEE Trans Med Imaging 23:137-152.

Beckmann CF, Smith SM (2005) Tensorial extensions of independent component analysis for multisubject FMRI analysis. Neuroimage 25:294-311.

Beckmann CF, Jenkinson M, Smith SM (2003) General multilevel linear modeling for group analysis in FMRI. Neuroimage 20:1052-1063.

Behzadi Y, Liu TT (2006) Caffeine reduces the initial dip in the visual BOLD response at $3 \mathrm{~T}$. Neuroimage 32:9-15.

Buschke H, Sliwinski MJ, Kuslansky G, Lipton RB (1997) Diagnosis of early dementia by the double memory test: encoding specificity improves diagnostic sensitivity and specificity. Neurology 48:989-997.

Cameron OG, Modell JG, Hariharan M (1990) Caffeine and human cerebral blood flow: a positron emission tomography study. Life Sci 47:1141-1146.

Costenla AR, Cunha RA, de Mendonca A (2010) Caffeine, adenosine receptors, and synaptic plasticity. J Alzheimers Dis 20(Suppl 1):S25-34.

Crunelle CL, Veltman DJ, Booij J, Emmerik-van Oortmerssen K, van den Brink W (2012) Substrates of neuropsychological functioning in stimulant dependence: a review of functional neuroimaging research. Brain Behav 2:499-523.

Cunha RA, Agostinho PM (2010) Chronic caffeine consumption prevents memory disturbance in different animal models of memory decline. J Alzheimers Dis 20(Suppl 1):S95-116.

D'Esposito M, Deouell LY, Gazzaley A (2003) Alterations in the BOLD fMRI signal with ageing and disease: a challenge for neuroimaging. Nat Rev Neurosci 4:863-872.

Diukova A, Ware J, Smith JE, Evans CJ, Murphy K, Rogers PJ, Wise RG (2012) Separating neural and vascular effects of caffeine using simultaneous EEG-FMRI: differential effects of caffeine on cognitive and sensorimotor brain responses. Neuroimage 62:239-249.

Dunwiddie TV, Masino SA (2001) The role and regulation of adenosine in the central nervous system. Annu Rev Neurosci 24:31-55.

Fazekas F, Chawluk JB, Alavi A, Hurtig HI, Zimmerman RA (1987) MR signal abnormalities at $1.5 \mathrm{~T}$ in Alzheimer's dementia and normal aging. AJR Am J Roentgenol 149:351-356.

Ferre S (2010) Role of the central ascending neurotransmitter systems in the psychostimulant effects of caffeine. J Alzheimers Dis 20(Suppl 1):S35-49. 
Fisone G, Borgkvist A, Usiello A (2004) Caffeine as a psychomotor stimulant: mechanism of action. Cell Mol Life Sci 61:857-872.

Folstein MF, Folstein SE, McHugh PR (1975) "Mini-mental state". A practical method for grading the cognitive state of patients for the clinician. J Psychiatr Res 12:189-198.

Fredholm BB, Battig K, Holmen J, Nehlig A, Zvartau EE (1999) Actions of caffeine in the brain with special reference to factors that contribute to its widespread use. Pharmacol Rev 51: 83-133.

Gauthier CJ, Madjar C, Desjardins-Crepeau L, Bellec P, Bherer L, Hoge RD (2013) Age dependence of hemodynamic response characteristics in human functional magnetic resonance imaging. Neurobiol Aging 34:1469-1485.

Griffeth VE, Perthen JE, Buxton RB (2011) Prospects for quantitative fMRI: investigating the effects of caffeine on baseline oxygen metabolism and the response to a visual stimulus in humans. Neuroimage 57:809-816.

Hughes CP, Berg L, Danziger WL, Coben LA, Martin RL (1982) A new clinical scale for the staging of dementia. $\mathrm{Br} J$ Psychiatry 140:566-572.

Hyvarinen A (1999) Fast and robust fixed-point algorithms for independent component analysis. IEEE Trans Neural Netw 10:626-634

lannetti GD, Wise RG (2007) BOLD functional MRI in disease and pharmacological studies: room for improvement? Magn Reson Imaging 25:978-988.

Kannurpatti SS, Motes MA, Rypma B, Biswal BB (2010) Neural and vascular variability and the $\mathrm{AMRI}-\mathrm{BOLD}$ response in normal aging. Magn Reson Imaging 28:466-476.

Kannurpatti SS, Motes MA, Rypma B, Biswal BB (2011) Increasing measurement accuracy of age-related BOLD signal change: minimizing vascular contributions by resting-state-fluctuation-ofamplitude scaling. Hum Brain Mapp 32:1125-1140.

Kaplan EF, Goodglass H, \& Weintraub S. The Boston naming test (2nd edition). In: Lea \& Febiger. Philadelphia: 1983.

Klaassen EB, de Groot RH, Evers EA, Snel J, Veerman EC, Ligtenberg AJ, Jolles J, Veltman DJ (2013) The effect of caffeine on working memory load-related brain activation in middle-aged males. Neuropharmacology 64:160-167.

Koppelstaetter F, Poeppel TD, Siedentopf CM, Ischebeck A, Verius M, Haala I, Mottaghy FM, Rhomberg P, Golaszewski S, Gotwald T, Lorenz IH, Kolbitsch C, Felber S, Krause BJ (2008) Does caffeine modulate verbal working memory processes? An fMRI study. Neuroimage 39:492-499.

Koppelstaetter F, Poeppel TD, Siedentopf CM, Ischebeck A, Kolbitsch C, Mottaghy FM, Felber SR, Jaschke WR, Krause BJ (2010) Caffeine and cognition in functional magnetic resonance imaging. J Alzheimers Dis 20(Suppl 1):S71-84.

Landau SM, Lal R, O'Neil JP, Baker S, Jagust WJ (2009) Striatal dopamine and working memory. Cereb Cortex 19:445-454.

Laurienti PJ, Field AS, Burdette JH, Maldjian JA, Yen Y-F, Moody DM (2002) Dietary caffeine consumption modulates fMRI measures. Neurolmage 17:751-757.

Laurienti PJ, Field AS, Burdette JH, Maldjian JA, Yen YF, Moody DM (2003) Relationship between caffeine-induced changes in resting cerebral perfusion and blood oxygenation level-dependent signal. AJNR Am J Neuroradiol 24:1607-1611.
Liu TT, Liau J (2010) Caffeine increases the linearity of the visual BOLD response. Neuroimage 49:2311-2317.

Liu TT, Behzadi Y, Restom K, Uludag K, Lu K, Buracas GT, Dubowitz DJ, Buxton RB (2004) Caffeine alters the temporal dynamics of the visual BOLD response. Neuroimage 23:1402-1413.

Milner B (1971) Interhemispheric differences in the localization of psychological processes in man. Br Med Bull 27:272-277.

Mulderink TA, Gitelman DR, Mesulam MM, Parrish TB (2002) On the use of caffeine as a contrast booster for BOLD fMRI studies. Neuroimage 15:37-44.

Owen AM, McMillan KM, Laird AR, Bullmore E (2005) N-back working memory paradigm: a meta-analysis of normative functional neuroimaging studies. Hum Brain Mapp 25:46-59.

Pelligrino DA, Xu HL, Vetri $F$ (2010) Caffeine and the control of cerebral hemodynamics. J Alzheimers Dis 20(Suppl 1):S51-62.

Perthen JE, Lansing AE, Liau J, Liu TT, Buxton RB (2008) Caffeineinduced uncoupling of cerebral blood flow and oxygen metabolism: a calibrated BOLD fMRI study. Neuroimage 40:237-247.

Rack-Gomer AL, Liu TT (2012) Caffeine increases the temporal variability of resting-state BOLD connectivity in the motor cortex. Neuroimage 59:2994-3002.

Rack-Gomer AL, Liau J, Liu TT (2009) Caffeine reduces resting-state BOLD functional connectivity in the motor cortex. Neuroimage 46:56-63.

Rees K, Allen D, Lader M (1999) The influences of age and caffeine on psychomotor and cognitive function. Psychopharmacology (Berl) 145:181-188.

Reitan RM (1958) Validity of the trail making test as an indicator of organic brain damage. Percept Mot Skills 8:271-276.

Rossi S, De Chiara V, Musella A, Mataluni G, Sacchetti L, Siracusano A, Bernardi G, Usiello A, Centonze D (2010) Effects of caffeine on striatal neurotransmission: focus on cannabinoid $\mathrm{CB} 1$ receptors. Mol Nutr Food Res 54:525-531.

Smith SM, Nichols TE (2009) Threshold-free cluster enhancement: addressing problems of smoothing, threshold dependence and localisation in cluster inference. Neuroimage 44:83-98.

Tal O, Diwakar M, Wong CW, Olafsson V, Lee R, Huang MX, Liu TT (2013) Caffeine-induced global reductions in resting-state BOLD connectivity reflect widespread decreases in MEG connectivity. Front Hum Neurosci 7:63.

Vidyasagar R, Greyling A, Draijer R, Corfield DR, Parkes LM (2013) The effect of black tea and caffeine on regional cerebral blood flow measured with arterial spin labeling. J Cereb Blood Flow Metab 33:963-968.

Wechsler D (1955) Manual for the Wechsler adult intelligence scale. New York: Psychological Corporation.

Wong CW, Olafsson V, Tal O, Liu TT (2012) Anti-correlated networks, global signal regression, and the effects of caffeine in resting-state functional MRI. Neuroimage 63:356-364.

Woolrich M (2008) Robust group analysis using outlier inference. Neuroimage 41:286-301.

Woolrich MW, Behrens TE, Beckmann CF, Jenkinson M, Smith SM (2004) Multilevel linear modelling for FMRI group analysis using Bayesian inference. Neuroimage 21:1732-1747.

Zigmond AS, Snaith RP (1983) The hospital anxiety and depression scale. Acta Psychiatr Scand 67:361-370. 